University of Nebraska - Lincoln

DigitalCommons@University of Nebraska - Lincoln

November 1999

\title{
Intensity dependence of plateau structures in laser-assisted x- ray-atom scattering processes
}

Dejan B. Miloševic

University of Nebraska - Lincoln

Anthony F. Starace

University of Nebraska-Lincoln, astarace1@unl.edu

Follow this and additional works at: https://digitalcommons.unl.edu/physicsstarace

Part of the Physics Commons

Miloševic, Dejan B. and Starace, Anthony F., "Intensity dependence of plateau structures in laser-assisted x-ray-atom scattering processes" (1999). Anthony F. Starace Publications. 71.

https://digitalcommons.unl.edu/physicsstarace/71

This Article is brought to you for free and open access by the Research Papers in Physics and Astronomy at DigitalCommons@University of Nebraska - Lincoln. It has been accepted for inclusion in Anthony F. Starace Publications by an authorized administrator of DigitalCommons@University of Nebraska - Lincoln. 


\title{
Intensity dependence of plateau structures in laser-assisted $x$-ray-atom scattering processes
}

\author{
Dejan B. Miloševic** and Anthony F. Starace \\ Department of Physics and Astronomy, The University of Nebraska, 116 Brace Laboratory, Lincoln, Nebraska 68588-0111
}

(Received 3 February 1999)

\begin{abstract}
The dependence on laser intensity of recently discovered plateau structures in laser-assisted x-ray-atom scattering, both with and without a static electric field present, is analyzed. Using the "three-step" model and the strong-field approximation we demonstrate a connection between laser-assisted, x-ray-atom scattering and high-order harmonic generation: For high laser-field intensities without a static field present, both processes have plateaus whose energies extend to the cutoff value $3.17 U_{p}$, where $U_{p}$ is the ponderomotive potential energy. For x-ray-atom scattering in the presence of a static electric field we show that at high laser-field intensities two plateaus appear: One is our recently predicted high-energy plateau for the same process [Phys. Rev. Lett. 81, 5097 (1998)], while the other, low-energy plateau, has a differential cross section six orders of magnitude larger. The energy positions and relative magnitudes of these new plateaus are explained using semiclassical arguments. [S1050-2947(99)07211-X]
\end{abstract}

PACS number(s): 32.80.Qk, 32.30.Rj, 34.80.Qb

Over the past two decades the behavior of atoms subjected to an intense electromagnetic field has been a subject of wide interest and active research [1-4]. The interaction of strong laser pulses and atoms results in a variety of phenomena that can only be explained using nonperturbative approaches. Among the many atomic processes in strong laser fields, special attention has been devoted to two processes: above-threshold ionization (ATI) and high-order harmonic generation (HHG). In both processes photons are absorbed from the laser field and their energy is transferred either to the photoelectron in the ATI process or to the harmonic photon in the HHG process. Recently, another atomic process in an intense laser field has received attention: laser-assisted x-ray-atom scattering both without [5] and with a strong static electric field [6]. In Ref. [5], plateaulike structures in the differential cross section (DCS) as a function of the number of photons $n$ exchanged with the laser field were found primarily for $n<0$ (i.e., emitted photons), indicating scattered $x$ rays having lower energies. It was shown in Ref. [6] that the addition of a static electric field gives rise to an extended plateau for $n>0$ (i.e., absorbed photons), indicating scattered $x$ rays having substantially higher energies. Coherent $x$ rays with energies in the "water window" from 284 to $532 \mathrm{eV}$ would have important applications for imaging living biological structures by means of x-ray holography [4]. We present here results showing a sensitive dependence of scattered x-ray plateau structures on laser field intensity, and demonstrate a connection between laser-assisted X-rayatom scattering and HHG.

The main characteristics of both the ATI and HHG processes can be explained using the so-called "two-" and "three-step" physical models (see, e.g., Kulander et al., p. 25 in Ref. [1], and Refs. [7,8]). As these models also allow one to interpret results for laser-assisted X-ray-atom scatter-

\footnotetext{
* On leave from: Faculty of Science and Mathematics, Department of Physics, University of Sarajevo, Zmaja od Bosne 35, 71000 Sarajevo, Bosnia and Herzegovina. Present address: Max-BornInstitut, Max-Born-Str. 2a, 12489 Berlin, Germany.
}

ing $[5,6]$, we discuss briefly their main features. The "first step" is the ionization of an atomic electron, while the "second step" is the propagation of a free electron in the laser field. Some of the characteristics of ATI can be explained using only these two steps. The "third step" is the collision between the electron, driven back by the laser field, and the atomic core, whereupon the electron can recombine with the ion, emitting a harmonic photon. This three-step model explains both the appearance of the plateau in the HHG process and the maximum energy of the harmonics at the cutoff $N_{\text {max }} \omega=I_{0}+3.17 U_{p}$, where $I_{0}$ is the atomic ionization potential, $U_{p}=E_{L}^{2} /\left(4 \omega^{2}\right)$ is the ponderomotive potential energy, and $E_{L}$ and $\omega$ are the laser electric-field amplitude and frequency, respectively. (We use here atomic + SI units.) Alternatively, during the third step the electron can scatter from the atomic core, giving rise to rescattering effects in ATI. In this case, the third step can explain the appearance of the plateau in ATI with its cutoff at $10 U_{p}$ [9]. The classical three-step model is consistent with results of quantummechanical calculations $[8,10,11]$. A key approximation in Refs. $[5,6,8,10,11]$ is the so-called strong-field approximation (SFA), in which the Green's propagator of the total system is replaced in intermediate states by the Volkov Green's propagator, i.e., the influence of the atomic potential on the electron is neglected in comparison with that of the laser field (and the static external fields if present, as in [6]). The SFA fails [12] if the number of photons exchanged with the laser field is small: for ATI this corresponds to photoelectrons with energies close to the threshold, while for HHG it corresponds to low-order harmonics. (For a comparison of results using the SFA with those obtained by solving the time-dependent, three-dimensional Schrödinger equation, see [12].) Analysis of these processes (i.e., ATI, HHG, and laserassisted $\mathrm{x}$-ray-atom scattering) starting from the appropriate quantum-mechanical amplitudes, applying the SFA, and evaluating the resulting amplitudes in a quasiclassical (stationary phase) approximation provides a more rigorous confirmation of the three-step model than does a purely classical calculation. From the above discussion, it is clear that ATI, HHG, and laser-assisted x-ray-atom scattering are related processes. 
Consider now the latter process. Using the SFA (which is appropriate when the number of photons $n$ exchanged with the laser field is large), the DCS for laser-assisted x-rayatom scattering with absorption $(n>0)$ or emission $(n<0)$ of $n$ laser photons is $[5,6]$

$$
\begin{gathered}
\frac{d \sigma(n)}{d \Omega_{\hat{\mathbf{K}}^{\prime}}}=c^{-4} \omega_{\mathbf{K}} \omega_{\mathbf{K}^{\prime}}^{3}\left|T_{\mathbf{K}, \mathbf{K}^{\prime}}^{(+)}(n)+T_{\mathbf{K}^{\prime}, \mathbf{K}^{(-)}}^{(n)}\right|^{2}, \\
T_{\mathbf{K}, \mathbf{K}^{\prime}}^{( \pm)}(n)=\int_{0}^{2 \pi} \frac{d \varphi}{2 \pi} \mathcal{T}_{\mathbf{K}, \mathbf{K}^{\prime}}^{( \pm)}(\varphi) \exp (i n \varphi),
\end{gathered}
$$

where $\omega_{\mathbf{K}}$ and $\omega_{\mathbf{K}^{\prime}}=\omega_{\mathbf{K}}+n \omega$ are the energies of the incident and scattered $\mathrm{x}$-ray photons, respectively, and

$$
\begin{aligned}
\mathcal{T}_{\mathbf{K}, \mathbf{K}^{\prime}}^{( \pm)}(\varphi)= & \int_{0}^{\infty} d \tau \int d \mathbf{q} \exp \left\{-i\left[S(\mathbf{q} ; t, \tau) \pm \omega_{\mathbf{K}^{\prime}} \tau\right]\right\} \\
& \times\left\langle\psi_{0}\left|\mathbf{r} \cdot \hat{\mathbf{e}}_{\mathbf{K}}\right| \mathbf{q}+\mathbf{A}(t)\right\rangle\left\langle\mathbf{q}+\mathbf{A}(t-\tau)\left|\mathbf{r} \cdot \hat{\mathbf{e}}_{\mathbf{K}^{\prime}}\right| \psi_{0}\right\rangle
\end{aligned}
$$

Here $\left|\psi_{0}\right\rangle$ is the ket vector of the atomic ground state; $|\mathbf{q}\rangle$ is the electron plane-wave ket vector; $\varphi=\omega t ; \hat{\mathbf{e}}_{\mathbf{K}}$ and $\hat{\mathbf{e}}_{\mathbf{K}^{\prime}}$ are the unit polarization vectors of the incident and scattered photons, respectively; $\mathbf{A}(t)$ is the vector potential for both a laser field and a static electric field; $S(\mathbf{q} ; t, \tau)=\int_{t-\tau}^{t} d t^{\prime}\left\{\frac{1}{2}[\mathbf{q}\right.$ $\left.\left.+\mathbf{A}\left(t^{\prime}\right)\right]^{2}+I_{0}\right\}$ is the electron's quasiclassical action; and $I_{0}=0.5$ a.u. is the ionization potential of the $\mathrm{H}$ atom, the same one considered in Refs. [5,6]. The matrix element $T_{\mathbf{K}^{\prime}, \mathbf{K}}^{(-)}(n)$ in Eq. (1) corresponds to the process in which an x-ray photon having wave vector $\mathbf{K}$ and energy $\omega_{\mathbf{K}}$ is absorbed first. The ionized electron propagates under the influence of both the laser field (and, if present, the static electric field) during the time interval from $t-\tau$ to $t$, at which time it returns to the atomic core (i.e., the return time is $\tau$ ). It then recombines with the atomic core, exchanging $n$ photons with the laser field and emitting an $\mathrm{x}$-ray photon having wave vector $\mathbf{K}^{\prime}$ and energy $\omega_{\mathbf{K}^{\prime}}$. The matrix element $T_{\mathbf{K}, \mathbf{K}^{\prime}}^{(+)}(n)$ describes the process in which the x-ray photon having wave vector $\mathbf{K}^{\prime}$ and energy $\omega_{\mathbf{K}^{\prime}}$ is emitted first. Reference [5] shows that the contribution of $T_{\mathbf{K}, \mathbf{K}^{\prime}}^{(+)}(n)$ to the DCS can be neglected in comparison to that of $T_{\mathbf{K}^{\prime}, \mathbf{K}}^{(-)}(n)$ (for $|n|>5$ ). The process described by $T_{\mathbf{K}^{\prime}, \mathbf{K}}^{(-)}(n)$ is in agreement with the three-step model for the HHG process except that during the "ionization step" the incident x-ray photon is absorbed. Details of the computation of the DCS are given in Ref. [5]. In brief, the three-dimensional integral over the intermediate electron momenta in Eq. (2) can be carried out using the time-dependent WBK approximation. The integral over the return time $\tau$ is computed numerically, and, finally, the $T$ matrices, given by Eq. (1), are obtained using the fast Fourier transform method. Explicit analytical forms of the matrix elements in Eq. (2) are given in Ref. [5] in terms of the vector potential $\mathbf{A}(t)$, the stationary momentum $\mathbf{q}_{s} \equiv \mathbf{q}_{s}(t, \tau)=-(1 / \tau) \int_{t-\tau}^{t} d t^{\prime} \mathbf{A}\left(t^{\prime}\right)$, and the stationary action $S_{s} \equiv S\left(\mathbf{q}_{s} ; t, \tau\right) . \mathbf{A}(t)$ corresponds to a linearly polarized electric-field vector $\mathbf{E}(t)=\left(E_{L} \sin \omega t+E_{S}\right) \hat{\mathbf{e}}$, where $\hat{\mathbf{e}}$ is the

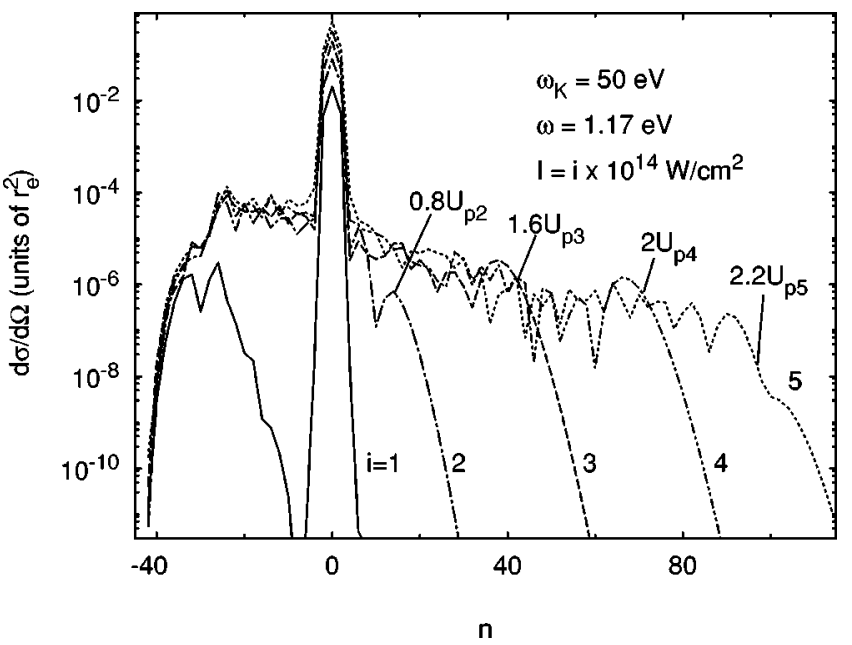

FIG. 1. The DCS for x-ray-hydrogen-atom scattering in units of $r_{e}^{2}$ (where $r_{e}=2.8 \times 10^{-15} \mathrm{~m}$ is the classical electron radius) as a function of the number $n$ of absorbed $(n>0)$ or emitted $(n<0)$ laser field photons, for different laser field intensities $I(i)$ $=i \times 10^{14} \mathrm{~W} / \mathrm{cm}^{2}$, where $i=1$ (solid curve), 2 (dot-dashed curve), 3 (dashed curve), 4 (double-dot-dashed curve), and 5 (dotted curve). The laser field is linearly polarized and monochromatic with photon energy $\omega=1.17 \mathrm{eV}$. The energy of the incident $\mathrm{x}$-ray photons is $\omega_{\mathbf{K}}=50 \mathrm{eV}$. The energy cutoff positions are denoted by multiples of the ponderomotive potential energy $U_{p i}$ for laser intensity $I(i)$.

unit polarization vector, and $E_{L}$ and $E_{S}$ are the amplitudes of the laser and static electric fields.

Consider first the case of $E_{S}=0$. In Ref. [5] numerical results for laser-assisted $\mathrm{x}$-ray-atom scattering were presented for a laser field intensity of less than $10^{14} \mathrm{~W} / \mathrm{cm}^{2}$. For an x-ray photon energy of $50 \mathrm{eV}$, a plateau was observed only for negative values of $n$. The question arises as to what will happen to the DCS if the laser field intensity is increased. We present our numerical results for a laser field having frequency $\omega=1.17 \mathrm{eV}$ and intensity $I \geqslant 10^{14} \mathrm{~W} / \mathrm{cm}^{2}$. The energy of the incident $\mathrm{x}$-ray photons is $\omega_{\mathbf{K}}=50 \mathrm{eV}$, and we consider only the case of parallel polarizations. Figure 1 shows the DCS for laser-assisted x-rayhydrogen-atom scattering as a function of the number $n$ of absorbed $(n>0)$ or emitted $(n<0)$ laser field photons for five different values of the laser field intensity $I$ between $10^{14}$ and $5 \times 10^{14} \mathrm{~W} / \mathrm{cm}^{2}$. A plateau for positive values of $n$ appears as $I$ increases, and, for the highest intensity, it is more than two times longer in $n$ than the plateau for negative values of $n$, which remains almost unchanged for $I \geqslant 2 \times 10^{14} \mathrm{~W} / \mathrm{cm}^{2}$. For positive values of $n$, the energy of the scattered $x$ rays is increased and the shape of the plateau vs $n$ is very similar to that for the HHG intensity, presented as a function of harmonic order $[10,12]$. For the HHG process, the cutoff of the plateau appears (for $U_{p} \gg I_{0}$ ) at $n_{\max } \omega \approx 3.17 U_{p}$, where $n_{\max }$ is the harmonic order. Figure 2 shows that this cutoff law is also valid for the laser-assisted $\mathrm{x}$-ray-atom-scattering process. Namely, we present there $n_{\max } \omega$ in units of $U_{p}$ as a function of the laser field intensity $I$, where now $n_{\max }$ is the number of absorbed laser photons. For large values of $I$, i.e., for large values of $U_{p}$, we observe that $n_{\max } \omega \rightarrow 3.17 U_{p}$ just as in the HHG process. A quasiclassical analysis [see Eq. (4) in Ref. [6]] shows that the proper cutoff law is $n_{\max } \omega=E_{k, \max }-\omega_{\mathbf{K}}+I_{0}$, where the maximum 


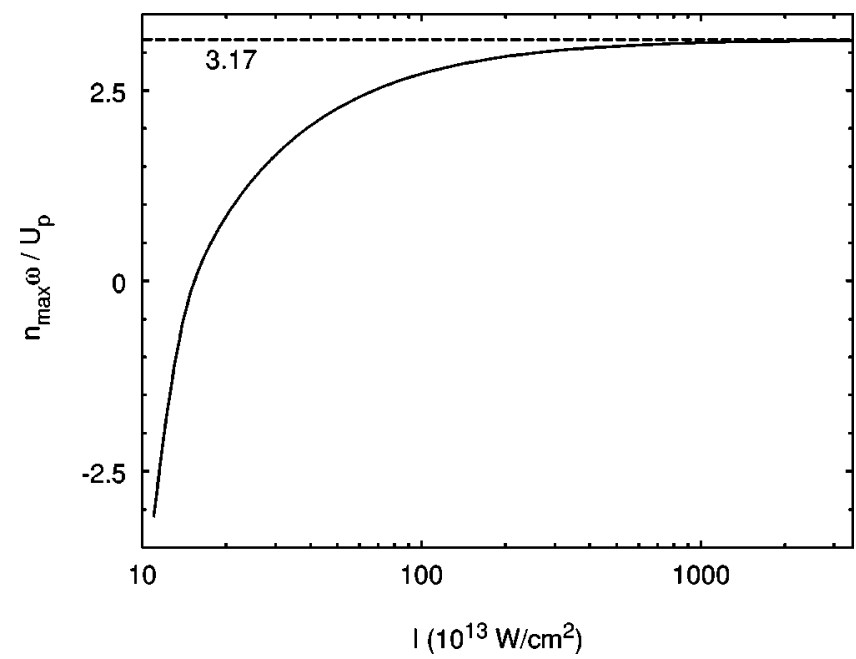

FIG. 2. Maximum energy exchanged with the laser field in laser-assisted x-ray-hydrogen-atom scattering, in units of the ponderomotive energy, as a function of the laser field intensity. The dashed line shows the $3.17 U_{p}$ cutoff of the HHG spectrum. The other parameters are as in Fig. 1.

kinetic energy that the electron can acquire in the laser field is $E_{k, \max }=3.17 U_{p}$. For $\mathrm{HHG}$, the cutoff formula for $n_{\max } \omega$ does not include a term $-\omega_{\mathbf{K}}$ because there are no X-ray photons in the initial state.

Consider now the addition of a static electric field having strength $E_{S}=2 \mathrm{MV} / \mathrm{cm}$, which is slightly less than the present maximum static field strength achieved experimentally [13], $3.5 \mathrm{MV} / \mathrm{cm}$. The laser field, atomic, and $\mathrm{x}$-ray parameters are as before. As in Ref. [6], the influence of the static electric field on the $\mathrm{H}$ atom ground state is neglected. (Recent results of Ivanov [14] indicate this approximation is valid up to $E_{S}=100 \mathrm{MV} / \mathrm{cm}$.) Figure 3 shows the DCS as a function of $n$ for a laser intensity $I(i)=i \times 10^{14} \mathrm{~W} / \mathrm{cm}^{2}$, where $i=1,3$, and 5. As in Ref. [6], a high-energy plateau appears. For $i=1$, this plateau extends up to $18.5 U_{p 1}(n$ 166), while for $i=3$ and $i=5$ the cutoffs are at $14.1 U_{p 3}(n=378)$ and $12.7 U_{p 5}(n=569)$, respectively,

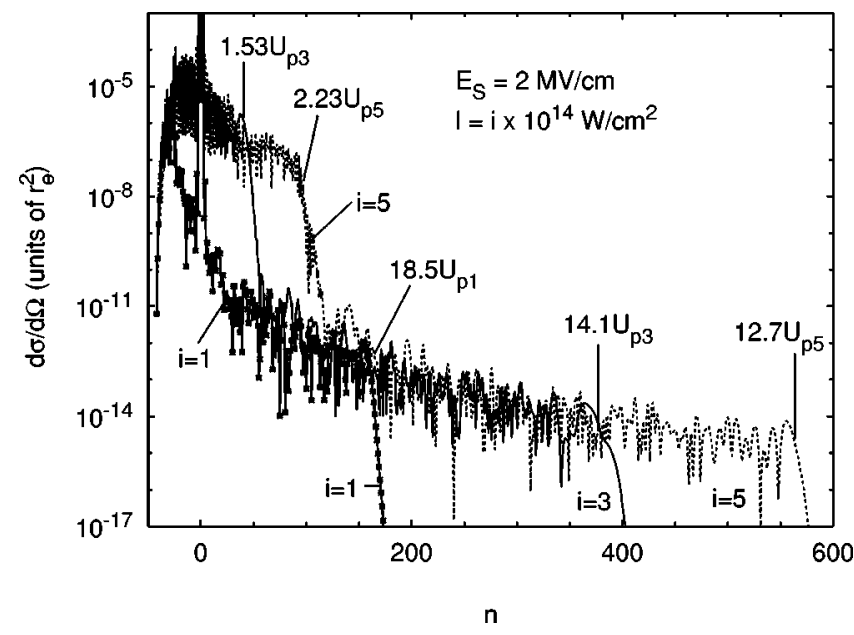

FIG. 3. The DCS in the presence of a static electric field, $E_{S}$ $=2 \mathrm{MV} / \mathrm{cm}$, presented as in Fig. 1. The laser field intensity is $I(i)=i \times 10^{14} \mathrm{~W} / \mathrm{cm}^{2}$, where $i=1$ (stars), 3 (solid curve), and 5 (dotted curve).

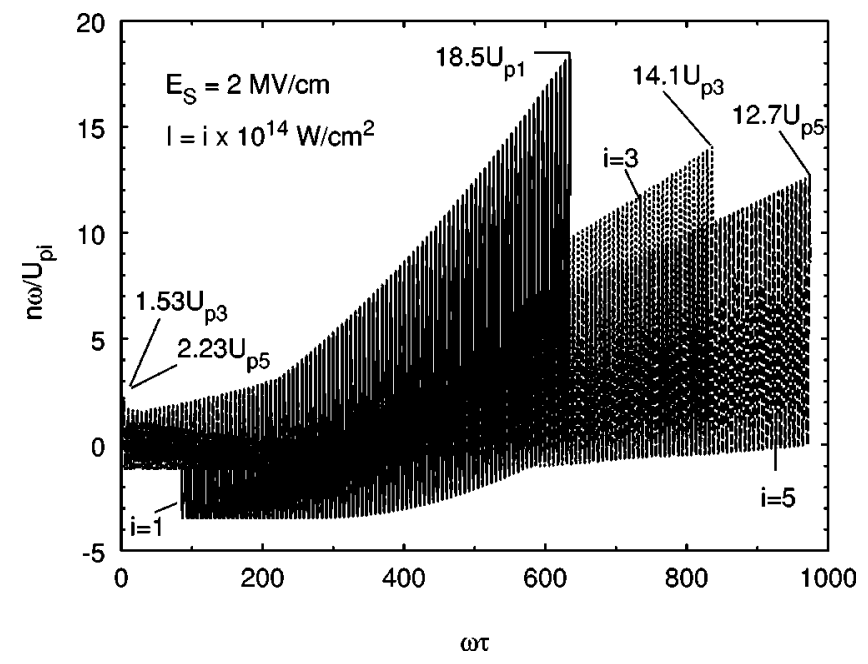

FIG. 4. Energy exchanged with the laser field in laser-assisted $\mathrm{X}$-ray-hydrogen-atom scattering in the presence of a static electric field $E_{S}=2 \mathrm{MV} / \mathrm{cm}$, in units of the ponderomotive energy $U_{p i}$, for laser intensities $I=i \times 10^{14} \mathrm{~W} / \mathrm{cm}^{2}$, where $i=1,3$, and 5 . Results are plotted vs the dimensionless variable $\omega \tau$, where $\tau$ is the electron return time to the origin under the influence of the laser and static fields as obtained by solving the semiclassical equations of Refs. [5,6]. The incident X-ray photon energy is $\omega_{\mathbf{K}}=50 \mathrm{eV}$. The energy maxima, expressed in multiples of $U_{p i}$, are denoted on each curve.

where $U_{p i}$ is the ponderomotive energy corresponding to the intensity $I(i)$. For $i=3$ and 5 additional plateaus appear for smaller values of $n$, with cutoffs at $1.53 U_{p 3}(n=41)$ and $2.23 U_{p 5}(n=100)$, respectively. The DCS's for these low $n$ plateaus are six orders of magnitude higher than those of the higher $n$ plateaus. Comparing with results for $E_{S}=0$ (cf. Fig. 1 for $i=1,3$, and 5), we conclude that these higher DCS plateaus for smaller $n$ have cutoffs that are approximately independent of $E_{S}$ for the values of $E_{S}$ employed here.

In order to interpret these plateau features, we use the semiclassical method of Ref. [6]. Figure 4 presents the energy exchanged with the laser field, $n \omega=\frac{1}{2}\left[\mathbf{q}_{s}\left(t_{1}, \tau\right)\right.$ $\left.+\mathbf{A}\left(t_{1}\right)\right]^{2}-\omega_{\mathbf{K}}+I_{0}$, in units of $U_{p i}$ (for $i=1,3$, and 5), as a function of the dimensionless variable $\omega \tau$, where $\tau$ is the return time of the ionized electron to the atomic core. All other parameters are as in Fig. 3. For each $I(i)$ there are two solutions of the quasiclassical equation for $n(\tau)$ (corresponding to whether $\mathbf{E}_{L}$ is parallel or antiparallel to $\mathbf{E}_{S}$ at the time of ionization); we present here only one solution since the two results are close to one another. For $i=1$, the electron's initial kinetic energy, $p_{0}^{2} / 2=\omega_{\mathbf{K}}-I_{0}$, is larger than the maximum energy which the electron can acquire in the laser field, so that the laser field alone is not strong enough to return the electron to the nucleus. Therefore, for $i=1$ there is no plateau for positive $n$ in Fig. 1. In the presence of a static field, the electron can be returned to the nucleus because the static field acts on it with the force $-E_{S} \hat{\mathbf{e}}$, and the electron kinetic energy increases with increasing return time $\tau$. But, as can be seen in Fig. 4, the solid $i=1$ curve has no solutions for $n \omega$ for $\omega \tau<85.4$. This is the reason why the positive $n$ plateau for smaller $n$ is absent in Fig. 3 for $i=1$. For larger values of $\omega \tau$, positive $n$ solutions exist and correspond to the appearance of a longer plateau for large values of $n$ (cf. Fig. 
3 ), whose cutoff at $18.5 U_{p 1}$ corresponds to the largest $\omega \tau$ $=\omega \tau_{m}=635$ for $i=1$ in Fig. 4. The kinetic energy that the electron acquires from the static field increases with an increase of the return time, and therefore for $\tau>\tau_{m}$ the laser field can no longer return the electron to the nucleus. The cutoffs for stronger laser fields $(i=3$ and $i=5)$ occur for even larger values of $\omega \tau_{m}$. These cutoff positions are in excellent agreement with those obtained by our quantummechanical calculations (cf. Fig. 3). The cutoff positions of the higher DCS plateaus at lower $n$ can also be explained using results presented in Fig. 4. For higher laser field intensities ( $i=3$ and $i=5)$, the function $n \omega(\omega \tau)$ has a pronounced maximum during the first optical cycle $(\omega \tau$ $<2 \pi$ ), followed by a lower maximum and increasing maxima in each subsequent optical cycle. This first maximum occurs for $n \omega=1.53 U_{p 3}$ and $2.23 U_{p 5}$ for $i=3$ and 5 , respectively (cf. Fig. 4). The cutoff positions of the higher DCS plateaus for smaller $n$ in Fig. 3 correspond to these maxima; the DCS's for these plateaus are higher than those for the plateaus for larger $n$ values because the electron's return time is shorter, so that spreading of the electron wave packet is less significant. For very high laser field intensities, the cutoff energies of the higher DCS plateaus at smaller $n$ increase toward the value $3.17 U_{p}$, while the cutoff energy, expressed in units of $U_{p i}$, of the lower DCS and higher $n$ plateaus decrease (cf. Figs. 3 and 4). For $I \rightarrow \infty, \omega \tau_{m} \rightarrow \infty$ and the magnitude of the high $n$ plateau goes to zero (owing to wave-packet spreading), so that we have only the cutoff at $3.17 U_{p}$, the same one as in HHG in the absence of a static electric field. However, if we increase both $E_{L}$ and $E_{S}$ while keeping the ratio $E_{S} / E_{L}$ fixed, then the cutoff of the lower $n$ plateau satisfies the relation $n_{\max } \omega \approx c_{1} U_{p}$, where $c_{1}$ is slightly larger than 3.17 . The increase comes from the energy which the electron acquires in the static electric field. For example, for $E_{S} / E_{L}=0.02, c_{1}=3.38$. For the higher $n$ plateau, the cutoff is at $n_{\max } \omega \approx c_{2} U_{p}$, where for $E_{S} / E_{L}$ $=0.02, c_{2} \approx 8$. These results are in agreement with those obtained by Lohr et al. [15] for HHG in a static electric field.

We have considered the intensity dependence of plateau structures in laser-assisted x-ray-atom scattering, both with and without a static electric field present. For high laser field intensities without a static field present, the DCS as a function of the energy exchanged with the laser field forms a plateau which extends up to $3.17 U_{p}$, which shows a clear connection between laser-assisted x-ray-atom scattering and HHG. If a static electric field is present, both high- and lowenergy plateaus appear. A quasiclassical explanation of the energy positions, as well as the magnitudes, of these plateaus has been presented.

This work has been supported in part by the National Science Foundation under Grant No. PHY-9722110.
[1] Super-Intense Laser-Atom Physics, edited by B. Piraux, A. L'Huillier, and K. Rzążewski, NATO Advanced Science Institutes Series, Series B: Physics Vol. 316 (Plenum, New York, 1993).

[2] Super-Intense Laser-Atom Physics IV, edited by H. G. Muller and M. V. Fedorov (Kluwer, Dordrecht, 1996).

[3] Multiphoton Processes 1996, edited by P. Lambropoulos and H. Walther, IOP Conf. Proc. No. 154 (Institute of Physics and Physical Society, Bristol, 1997).

[4] C. Spielmann, N. H. Burnett, S. Sartania, R. Koppitsch, M. Schnürer, C. Kan, M. Lenzner, P. Wobrauschek, and F. Krausz, Science 278, 661 (1997).

[5] D. B. Milošević and F. Ehlotzky, Phys. Rev. A 58, 2319 (1998).

[6] D. B. Milošević and A. F. Starace, Phys. Rev. Lett. 81, 5097 (1998).

[7] P. B. Corkum, Phys. Rev. Lett. 71, 1994 (1993).

[8] W. Becker, A. Lohr, and M. Kleber, Quantum Semiclassic. Opt. 7, 423 (1995).
[9] G. G. Paulus, W. Becker, W. Nicklich, and H. Walther, J. Phys. B 27, L703 (1994).

[10] A. L'Huillier, M. Lewenstein, P. Salières, Ph. Balcou, M. Yu. Ivanov, J. Larsson, and C. G. Wahlstrom, Phys. Rev. A 48, R3433 (1993); M. Lewenstein, Ph. Balcou, M. Yu. Ivanov, A. L'Huillier, and P. B. Corkum, ibid. 49, 2117 (1994); D. B. Milošević and B. Piraux, ibid. 54, 1522 (1996).

[11] M. Lewenstein, K. C. Kulander, K. J. Schafer, and P. H. Bucksbaum, Phys. Rev. A 51, 1495 (1995); A. Lohr, M. Kleber, R. Kopold, and W. Becker, ibid. 55, R4003 (1997); D. B. Milošević and F. Ehlotzky, ibid. 57, 5002 (1998); 58, 3124 (1998).

[12] Ph. Antoine, B. Piraux, D. B. Milošević, and M. Gajda, Laser Phys. 7, 594 (1997).

[13] T. Bergeman et al., Phys. Rev. Lett. 53, 775 (1984); W. W. Smith et al., in Atomic Excitation and Recombination in External Fields, edited by M. H. Nayfeh and C. W. Clark (Gordon and Breach, New York, 1985), p. 211.

[14] M. V. Ivanov, J. Phys. B 31, 2833 (1998).

[15] A. Lohr, W. Becker, and M. Kleber, Laser Phys. 7, 615 (1997). 